\title{
REPRESENTATION STACKS, D-BRANES AND NONCOMMUTATIVE GEOMETRY
}

\author{
LIEVEN LE BRUYN
}

\begin{abstract}
In this note we prove that the $\operatorname{spec}(C)$-points of the representation Artin-stack $\left[\operatorname{rep}_{n} R / P G L_{n}\right]$ of $n$-dimensional representations of an affine $\mathbb{C}$ algebra $R$ correspond to $\mathbb{C}$-algebra morphisms $R \longrightarrow A_{n}$ where $A_{n}$ is an Azumaya algebra of degree $n$ over $C$. We connect this to the theory of Dbranes and Azumaya noncommutative geometry, developed by Chien-Hao Liu and Shing-Tung Yau in a series of papers [9]-14].
\end{abstract}

\section{REPRESENTATion STACKS}

Throughout, all algebras will be associative, unital $\mathbb{C}$-algebras which are finitely generated, that is, have a presentation

$$
R=\frac{\mathbb{C}\left\langle x_{1}, \ldots, x_{k}\right\rangle}{\left(p_{i}\left(x_{1}, \ldots, x_{k}\right) \mid i \in I\right)}
$$

With $\operatorname{rep}_{n} R$ we will denote the affine scheme of all $n$-dimensional representations of $R$. Its coordinate ring is the quotient of the polynomial algebra in all entries of $k$ generic $n \times n$ matrices

$$
X_{i}=\left[\begin{array}{ccc}
x_{11}(i) & \ldots & x_{1 n}(i) \\
\vdots & & \vdots \\
x_{n 1}(i) & \ldots & x_{n n}(i)
\end{array}\right]
$$

modulo the ideal generated by all the matrix-entries of the $n \times n$ matrices $p_{i}\left(X_{1}, \ldots, X_{k}\right)$ for all $i \in I$. That is,

$$
\mathcal{O}\left(\operatorname{rep}_{n} R\right)=\frac{\mathbb{C}\left[x_{i j}(l): 1 \leq i, j \leq n, 1 \leq l \leq k\right]}{\left(p_{i}\left(X_{1}, \ldots, X_{k}\right)_{u v}: 1 \leq u, v \leq n, i \in I\right)}
$$

The affine group scheme $G L_{n}$ (or rather $P G L_{n}$ ) acts via simultaneous conjugation on the generic matrices $X_{i}$ and hence on the affine scheme $\operatorname{rep}_{n} R$, its orbits corresponding to isomorphism classes of $n$-dimensional representations of $R$. It is well-known that the GIT-quotient $\operatorname{rep}_{n} R / P G L_{n}$, that is the affine scheme corresponding to the ring of polynomial invariants $\mathcal{O}\left(\operatorname{rep}_{n} R\right)^{P G L_{n}}$, classifies isomorphism classes of semi-simple $n$-dimensional representations of $R$, see [18] or [7, Chp. 2].

In order to investigate all orbits, one considers the $n$-dimensional representation stack $\left[\operatorname{rep}_{n} R / P G L_{n}\right]$. By this we mean (following 2]) the category with objects all triples $(Z, P, \phi)$ where $Z$ a scheme over $\mathbb{C}, \pi_{P}: P \longrightarrow Z$ a $P G L_{n}$-torsor in the 
étale topology

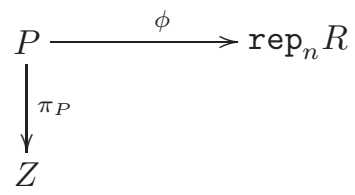

and with $\phi$ a $P G L_{n}$-equivariant map. Morphisms in this category are pairs $(f, h)$ : $(Z, P, \phi) \longrightarrow\left(Z^{\prime}, P^{\prime}, \phi^{\prime}\right)$ where $f: Z \longrightarrow Z^{\prime}$ is a morphism

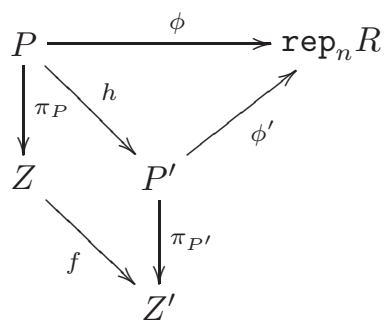

and $h$ is a $P G L_{n}$-equivariant map such that $P \simeq Z \times Z_{Z^{\prime}} P^{\prime}$ and $\phi=\phi^{\prime} \circ h$.

The full subcategory consisting of all triples $(Z, P, \phi)$ with fixed $Z$ are called the $Z$-points of the stack and are denoted by $\left[\operatorname{rep}_{n} R / P G L_{n}\right](Z)$. Observe that this subcategory is a groupoid, that is, all its morphisms are isomorphisms.

Theorem 1. For a commutative affine $\mathbb{C}$-algebra $C$, the $\operatorname{spec}(C)$-points of the representation stack $\left[\operatorname{rep}_{n} R / P G L_{n}\right]$ are in natural one-to-one correspondence with $\mathbb{C}$-algebra morphisms $R \longrightarrow A_{n}$ where $A_{n}$ is an Azumaya algebra of degree $n$ with center $C$.

Before we can prove this result, we need to recall some facts on Cayley-Hamilton algebras, see [18] and [7, and on Azumaya algebras, see [3] and [5].

Every $\mathbb{C}$-algebra $R$ has a universal trace map $n_{R}: R \longrightarrow R /[R, R]_{v}$ where $[R, R]_{v}$ is the sub-vectorspace of $R$ spanned by all commutators $[r, s]=r s-s r$ in $R$. It allows us to define the necklace functor

$$
\oint: \text { alg } \longrightarrow \text { commalg }
$$

which assign to any $\mathbb{C}$-algebra $R$ its necklace algebra $\oint R=\operatorname{Sym}\left(\frac{R}{[R, R]_{v}}\right)$ where for any $\mathbb{C}$-vectorspace $V$ we denote by $\operatorname{Sym}(V)$ the symmetric algebra on $V$.

With alg@ we denote the category of $\mathbb{C}$-algebras with trace. That is, a $\mathbb{C}$-algebra $R$ belongs to alg@ if it has a linear map $t r: R \longrightarrow R$ satisfying the following properties for all $r, s \in R$

$$
\left\{\begin{array}{l}
\operatorname{tr}(r) s=s t r(r) \\
\operatorname{tr}(r s)=\operatorname{tr}(s r) \\
\operatorname{tr}(\operatorname{tr}(r) s)=\operatorname{tr}(r) \operatorname{tr}(s)
\end{array}\right.
$$

In particular, it follows that the image of the trace map is contained in the center $Z(R)$. Morphisms in alg@ are trace preserving $\mathbb{C}$-algebra morphisms. The forgetful functor alg@ $\longleftrightarrow$ alg has a left adjoint, called the trace algebra functor

$$
\int: \operatorname{alg} \longrightarrow \operatorname{alg} @ \quad R \mapsto \int R=\oint R \otimes R
$$


with the trace map on $\int R$ defined by $\operatorname{tr}(c \otimes r)=c n_{R}(r) \otimes 1$, see [7. That is, for any $\mathbb{C}$-algebra $R$ and any $\mathbb{C}$-algebra with trace $A$, there is a natural one-to-one correspondence

$$
H_{\mathrm{alg} \Theta}\left(\int R, A\right) \longrightarrow \operatorname{Hom}_{\mathrm{alg}}(R, A) \quad \text { given by } \quad f \mapsto f \circ n_{R}
$$

Let $M \in M_{n}(\mathbb{C})$ be a diagonalizable matrix with distinct eigenvalues $\left\{\lambda_{1}, \ldots, \lambda_{n}\right\}$, then the characteristic polynomial has as coefficients the elementary symmetric functions $\sigma_{i}=\sigma_{i}\left(\lambda_{1}, \ldots, \lambda_{n}\right)$. Another generating set of the ring of symmetric functions is given by the power sums $s_{i}=\lambda_{1}^{i}+\ldots+\lambda_{n}^{i}$, hence there exist uniquely determined polynomials $p_{i}$ such that

$$
\sigma_{i}\left(\lambda_{1}, \ldots, \lambda_{n}\right)=p_{i}\left(s_{1}, \ldots, s_{n}\right)=p_{i}\left(\operatorname{Tr}(M), \ldots, \operatorname{Tr}\left(M^{i}\right)\right)
$$

because $s_{i}=\operatorname{Tr}\left(M^{i}\right)$.

This allows us to define for an algebra with trace $\left(R, t r_{R}\right) \in$ alg@ the formal Cayley-Hamilton polynomial of degree $n$ for all elements $a \in R$ by

$\chi_{a}^{(n)}(t)=t^{n}+p_{1}\left(\operatorname{tr}_{R}(a)\right) t^{n-1}+p_{2}\left(\operatorname{tr}_{R}(a), \operatorname{tr}_{R}\left(a^{2}\right)\right) t^{n-2}+\ldots+p_{n}\left(\operatorname{tr}_{R}(a), \ldots, t r_{R}\left(a^{n}\right)\right)$

With alg@n we denote the category of all Cayley-Hamilton algebras of degree n, that is, having as its objects algebras with trace map $\left(R, \operatorname{tr}_{R}\right)$ satisfying $\operatorname{tr}_{R}(1)=n$ and $\chi_{a}^{(n)}(a)=0$ for all $a \in R$, and, trace preserving $\mathbb{C}$-algebra maps as morphisms. We have functors

$$
\left\{\begin{array}{lll}
\int_{n}: \text { alg } \longrightarrow \text { alg@n } & R \mapsto \frac{\int R}{\left(\operatorname{tr}_{R}(1)-n, \chi_{a}^{(n)}(a) \forall a \in \int R\right)} \\
\oint_{n}: \text { alg } \longrightarrow \text { commalg } & R \mapsto t \int_{\int_{n}} R\left(\int_{n} R\right)
\end{array}\right.
$$

The main structural results on the trace algebras $\int_{n} R$ and $\oint_{n} R$ and their invariant theoretic interpretation are summarized in the next theorem, due to Claudio Procesi. For a proof and more details the reader is referred to [7, Chp. 2] or to the original paper [18.

Theorem 2 (Procesi). Let $R$ be a finitely generated $\mathbb{C}$-algebra. Then, with notations as before

(1) $\oint_{n} R$ is an affine commutative $\mathbb{C}$-algebra

(2) $\int_{n} R$ is a finitely generated module over $\oint_{n} R$

(3) $\oint_{n} R$ is the coordinate ring of the GIT-quotient scheme $\operatorname{rep}_{n} R / P G L_{n}$

(4) $\int_{n} R$ is the ring of all $P G L_{n}$-equivariant maps $\operatorname{rep}_{n} R \longrightarrow M_{n}(\mathbb{C})$

Azumaya algebras form an important class of Cayley-Hamilton algebras. For a $C$-algebra $R$ denote its enveloping algebra $R^{e}=R \otimes_{C} R^{o p}$, where $R^{o p}$ is the $C$ algebra with opposite multiplication map. There is a natural $C$-algebra morphism

$$
j_{R}: R^{e}=R \otimes_{C} R^{o p} \longrightarrow \operatorname{End}_{C}(R) \quad j_{R}(a \otimes b) r=a r b
$$

Definition 1. A $C$-algebra $A$ is called an Azumaya algebra iff $A$ is a finitely generated projective $C$-module and the map $j_{A}$ is an isomorphism of $C$-algebras.

It follows that the center $Z(A)$ of $A$ is equal to $C$ and that $A / \mathfrak{m} A \simeq M_{n}(\mathbb{C})$ for every maximal ideal $\mathfrak{m}$ of $C$ where $n^{2}$ is the local rank of $A$ at $\mathfrak{m}$. If the local rank is constant and equal to $n^{2}$ we say that $A$ is an Azumaya algebra of degree $n$. We recall the geometry associated to $C$-Azumaya algebras of degree $n$. 
Lemma 1. $C$-Azumaya algebras of degree $n$ are classified up to $C$-algebra isomorphism by the étale cohomology group $H_{\text {et }}^{1}\left(\operatorname{spec}(C), P G L_{n}\right)$, that is, C-Azumaya algebras of degree $n$ correspond to principal $P G L_{n}$-fibrations over $\operatorname{spec}(C)$.

Proof. It is well known (see for example [5]) that $A$ is a $C$-Azumaya algebra of degree $n$ if and only if there exist étale extensions $C \longrightarrow D$ splitting $A$, that is, such that $A \otimes_{C} D \simeq M_{n}(D)$. As $A u t\left(M_{n}(D)\right)=P G L_{n}(D)$, the isoclasses of such algebras are classified by the claimed cohomology group, see for example [16].

The principal $P G L_{n}$-fibration corresponding to the degree $n C$-Azumaya algebra $A$ is the representation scheme

$$
\operatorname{rep}_{n} A \longrightarrow \operatorname{rep}_{n} A / P G L_{n}=\operatorname{spec}(C)
$$

as all finite dimensional simple $A$-representation have to be $n$-dimensional because $A / \mathfrak{m} A=M_{n}(\mathbb{C})$. By étale descent, $A$ has a reduced trace map with $\operatorname{tr}(A)=C$, and hence we deduce from theorem 2 that $A \in$ alg@n and

$$
\int_{n} A=A \quad \text { and } \quad \oint_{n} A=C
$$

We have now all the tools needed to prove theorem 1.

Proof of theorem 1: Every $\mathbb{C}$-algebra morphism $\beta: R \longrightarrow A_{n}$ induces a $P G L_{n}$-equivariant map

$$
\beta^{*}: \operatorname{rep}_{n} A_{n} \longrightarrow \operatorname{rep}_{n} R
$$

by composition. By the above remarks we know that the GIT-quotient

$$
\operatorname{rep}_{n} A_{n} \stackrel{\pi}{\longrightarrow} \operatorname{rep}_{n} A_{n} / P G L_{n}=\operatorname{spec}(C)
$$

is a $P G L_{n}$-torsor. Therefore $\beta: R \longrightarrow A_{n}$ determines the $\operatorname{spec}(C)$-point of the representation stack

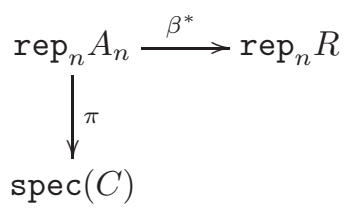

Conversely, $P G L_{n}$-torsors over $\operatorname{spec}(C)$ are classified by the pointed set

$$
H_{e t}^{1}\left(\operatorname{spec}(C), P G L_{n}\right)
$$

which also classifies the isomorphism classes of Azumaya algebras of degree $n$ with center $C$, see for example [16, p. 134]. Hence, any spec $(C)$-point of the representation stack $\left[\operatorname{rep}_{n} R / P G L_{n}\right]$ is of the form

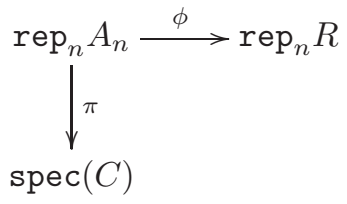

Taking $P G L_{n}$-equivariant maps to $M_{n}(\mathbb{C})$ on both sides of the $P G L_{n}$-equivariant map $\phi$ gives us by theorem 2 and the remarks above a trace preserving algebra 
morphism

$$
\int_{n} R \stackrel{\phi_{*}}{\longrightarrow} \int_{n} A_{n}=A_{n}
$$

and composing this with the universal morphism $R \longrightarrow \int_{n} R$ we obtain the desired $\mathbb{C}$-algebra morphism $R \stackrel{\beta}{\longrightarrow} A_{n}$ which induces $\phi=\beta^{*}$.

\section{Algebraic D-Branes}

In this section we relate the above to the description of D-branes via Azumaya noncommutative geometry as developed by Chian-Hao Liu and Shing-Tung Yau in [9- 14].

Let the affine variety $X$ be an affine open piece of (a spatial slice of) space-time and let $Z$ be a closed subscheme giving the boundary conditions for the endpoints of open strings (a D-brane). Wrapping one brane around $Z$ gives a $\mathbb{C}^{*}$-bundle (or a $U(1)$-symmetry) and the embedding of the D-brane in space-time $X$ corresponds to the quotient map of the corresponding coordinate rings

$$
\beta_{1}: \mathcal{O}(X) \longrightarrow \mathcal{O}(Z)
$$

However, if there are $n$ branes wrapped around $Z$, then $Z$ comes equipped with a $G L_{n}$-bundle $P$ (or a $U(n)$-symmetry) and the so called Polchinski Ansatz, see for example 9], asserts that the embedding of the $n$-stack of branes in space time is now governed by a $\mathbb{C}$-algebra morphism

$$
\beta: \mathcal{O}(X) \longrightarrow \operatorname{End}_{\mathcal{O}(Z)}(P)
$$

where $P$ is the so-called Chan-Patton bundle on $Z$. Observe that $\operatorname{End}_{\mathcal{O}(Z)}(P)$ is a trivial Azumaya algebra of degree $n$ with center $\mathcal{O}(Z)$ and hence the embedding morphism $\beta$ gives a $Z$-point of the representation stack $\left[\operatorname{rep}_{n} \mathcal{O}(X) / P G L_{n}\right]$.

In more general situations, for example when the $B$-field is turned on, one may replace the trivial Azumaya algebra $\operatorname{End}_{\mathcal{O}(Z)}(P)$ by a non-trivial Azumaya algebra $A_{n}$ of degree $n$ with center $\mathcal{O}(Z)$. Sometimes, one even allows for a noncommutative space time $R$. Motivated by this, we formalize D-branes in a purely algebraic context.

Definition 2. For $R$ an affine $\mathbb{C}$-algebra and $Z$ an affine scheme we will call a $Z$-point of the representation stack

$$
\beta \in\left[\operatorname{rep}_{n} R / P G L_{n}\right](Z)
$$

an algebraic D-brane of degree $n$ on $Z$.

The upshot of this interpretation of a D-brane embedding as a $Z$-point in the representation stack $\left[\operatorname{rep}_{n} \mathcal{O}(X) / P G L_{n}\right]$ (or more generally $\left[\operatorname{rep}_{n} R / P G L_{n}\right]$ ) is that one expects good properties of D-branes whenever the representation stack is smooth, in particular when $\operatorname{rep}_{n} R$ is a smooth affine variety.

This connects the study of D-branes to that of Cayley-smooth orders and formally smooth algebras as described in detail in the book [7. Observe that several of the examples worked out by Liu and Yau, such as the case of curves in [9, §4] and [10, or the case of the conifold algebra [12] (see also 8]) fall in this framework.

The dynamical aspect of D-branes, that is their Higgsing and un-Higgsing behavior as in [9] can also be formalized purely algebraically by enhancing the category alg of all $\mathbb{C}$-algebras with a specific class of 2 -morphisms. 
Definition 3. A 2-morphism between two $\mathbb{C}$-algebra morphisms $f$ and $g$

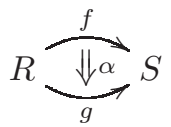

is a $Z(S)$-algebra mono-morphism between the centralizers in $S$ of the images

$$
\alpha: C_{S}(\operatorname{Im}(f)) \hookrightarrow C_{S}(\operatorname{Im}(g))
$$

If such a 2-morphism exists we say that $f$ degenerates to $g$ or, equivalently, that $g$ deforms to $f$.

In the case of algebraic D-branes, that is when $S=A_{n}$ is an Azumaya algebra of degree $n$ with center $C=\mathcal{O}(Z)$, these 2-morphisms correspond to the notions of 'Higgsing' and 'un-Higgsing' as in [9]. Here, the idea is that the Lie-algebra structure of the centralizer $C_{A}(\operatorname{Im}(\beta))$ can be interpreted as the gauge-symmetry group of the D-brane. Higgsing corresponds to symmetry-breaking, that is the gauge-group becomes smaller or, in algebraic terms, a 2-morphism deformation. Likewise, un-Higgsing corresponds to a 2-morphism degeneration of the algebraic D-brane.

Example 1. There are two extremal cases of algebraic D-branes. The 'maximal' ones coming from epimorphisms

$$
R \stackrel{\beta}{\longrightarrow} A
$$

If $C$ is the center of $A$ such a $D$-brane determines (and is determined by) a $\operatorname{spec}(C)$ family of simple $n$-dimensional representations of $R$. At the other extreme, 'minimal' algebraic D-branes are given by a composition

$$
\beta: R \stackrel{\pi}{\longrightarrow} C \stackrel{i}{\longrightarrow} A
$$

where $C$ is a commutative quotient of $R$ and $A$ is an Azumaya algebra over $C$. Such D-branes essentially determine a $\operatorname{spec}(C)$-family of one-dimensional representations of $R$.

For a maximal D-brane $\beta_{\max }: R \longrightarrow A$ we have $C_{A}\left(\operatorname{Im}\left(\beta_{\max }\right)\right)=Z(A)$ whence for any other algebraic $D$-brane $g: R \longrightarrow A$ we have a two-cell and hence a degeneration

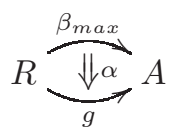

Likewise, if $\beta_{\text {min }}: R \longrightarrow C \longrightarrow A$ is a minimal D-brane we have $C_{A}\left(\operatorname{Im}\left(\beta_{\min }\right)\right)=A$ whence for any other D-brane we have a two-cell and corresponding deformation

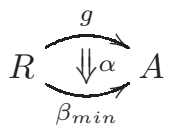

Note however that these 2-morphisms do not equip alg with a 2-category structure as defined for example in [15, XII.3]. Indeed, whereas we have an obvious vertical composition of 2-morphisms there is in general no horizontal composition of 2-morphisms. 
Still, these 2-morphisms impose a natural compatibility structure on families of algebraic D-branes. In string-theory one often considers the limit $n \rightarrow \infty$ of $\mathrm{n}$ stacks of D-branes located at a subscheme $Z \longrightarrow X$, that is, a family of algebra morphisms $\beta_{n}: \mathbb{C}[X] \longrightarrow A_{n}$ where $A_{n}$ is a degree $n$ Azumaya algebra with center $\mathbb{C}[Z]$. Our ringtheoretical considerations suggest one needs to impose a multiplicative compatibility requirement on such families.

Definition 4. A family of algebraic D-branes

$$
\beta_{n}: R \longrightarrow A_{n}
$$

such that $Z\left(A_{n}\right)=C$ for all $n$ is said to be compatible if for every $n \mid m$ we have a C-monomorphism $i_{n m}: A_{n} \longrightarrow A_{m}$ and a corresponding 2-morphism

$$
R \underset{i_{n m} \circ \beta_{n}}{\stackrel{\beta_{m}}{\Downarrow^{\alpha}}} A_{m}
$$

That is, the algebraic D-brane $\beta_{m}$ corresponding to the $m$ stack of D-branes at $\operatorname{spec}(C)$ is a Higgsing of all D-branes $R \longrightarrow A_{n} \longrightarrow A_{m}$ for all divisors $n$ of $m$.

We will give an example of a non-trivial example of a family of algebraic Dbranes which are all neither minimal nor maximal. Consider the 2-dimensional torus $\mathbb{C}\left[s^{ \pm}, t^{ \pm}\right]$and a primitive $n$-th root of unity $q_{n}=\sqrt[n]{1}$. The quantum torus is the non-commutative algebra generated by two elements $U_{n}$ and $V_{n}$ (and their inverses) satisfying the relations

$$
V_{n} U_{n}=q_{n} U_{n} V_{n} \quad U_{n}^{n}=s \quad V_{n}^{n}=t
$$

and we will denote this algebra as $\mathbb{C}_{q_{n}}\left[U_{n}^{ \pm}, V_{n}^{ \pm}\right]$. The center of this algebra is easily seen to be $\mathbb{C}\left[s^{ \pm}, t^{ \pm}\right]$and in fact $\mathbb{C}_{q_{n}}\left[U_{n}^{ \pm}, V_{n}^{ \pm}\right]$is an Azumaya algebra over it of degree $n$. When $m=n . k$ there are obvious embeddings of $\mathbb{C}\left[s^{ \pm}, t^{ \pm}\right]$-algebras

$$
i_{m, n}: \mathbb{C}_{q_{n}}\left[U_{n}^{ \pm}, V_{n}^{ \pm}\right] \hookrightarrow \mathbb{C}_{q_{m}}\left[U_{m}^{ \pm}, V_{m}^{ \pm}\right] \quad\left\{\begin{array}{l}
U_{n} \longrightarrow U_{m}^{k} \\
V_{n} \longrightarrow V_{m}^{k}
\end{array}\right.
$$

Lemma 2. Consider the coordinate ring of $G L_{2}=\left[\begin{array}{ll}s & u \\ v & t\end{array}\right]$

$$
\mathcal{O}\left(G L_{2}\right)=\mathbb{C}\left[s, t, u, v,(s t-u v)^{-1}\right]
$$

then the $\mathbb{C}$-algebra morphisms $\beta_{n}: \mathcal{O}\left(G L_{2}\right) \longrightarrow \mathbb{C}_{q_{n}}\left[U_{n}^{ \pm}, V_{n}^{ \pm}\right]$defined by

$$
\beta=\left(\beta_{n}\right):\left\{\begin{array}{l}
u \mapsto 0 \\
v \mapsto 0 \\
s \mapsto s \\
t \mapsto V_{n}
\end{array}\right.
$$

is a compatible family of algebraic D-branes on the maximal torus $T_{2} \longrightarrow G L_{2}$.

Proof. Clearly the maps $\beta_{n}$ are $\mathbb{C}$-algebra morphisms from $\mathcal{O}\left(G L_{2}\right)$ to the Azumaya algebra $\mathbb{C}_{q_{n}}\left[U_{n}^{ \pm}, V_{n}^{ \pm}\right]$whence they are algebraic D-branes. Remains for every $m=$ $n . k$ to compare the centralizers of the images at level $m$ with those of the image with the inclusion $i_{m, n}$. One easily verifies that

$$
\operatorname{Im}\left(\beta_{m}\right)=\mathbb{C}\left[s^{ \pm}, V_{m}^{ \pm}\right] \quad \text { whereas } \operatorname{Im}\left(i_{m, n} \circ \beta_{n}\right)=\mathbb{C}\left[s^{ \pm}, V_{m}^{ \pm k}\right]
$$


The centralizer of $\operatorname{Im}\left(\beta_{m}\right)$ is equal to itself, whereas

$$
C\left(\operatorname{Im}\left(i_{m, n} \circ \beta_{n}\right)=\mathbb{C}\left[s^{ \pm}, V_{m}^{ \pm k}\right] \otimes_{\mathbb{C}\left[s^{ \pm}, t^{ \pm}\right]} \mathbb{C}_{q_{k}}\left[U_{m}^{ \pm n}, V_{m}^{ \pm n}\right]\right.
$$

and so these form indeed a compatible family of algebraic D-branes on $T_{2}$.

\section{Azumaya noncommutative Geometry}

The main problem studied in the papers 9-14 by Chien-Hao Liu and ShingTung Yau is to associate some noncommutative geometry to the morphism $\beta$ : $\mathcal{O}(X) \longrightarrow A_{n}$ (or more generally $\beta: R \longrightarrow A_{n}$ ) describing the embedding of the n-stack of D-branes in space-time. That is, one wants to associate geometric objects (such as varieties, topological spaces and sheaves on these) that will allow us to reconstruct $\beta$ from the geometric data.

3.1. Noncommutative structure sheaves. We will rephrase Liu and Yau's Azumaya noncommutative geometry, based on 'surrogates' and the 'noncommutative cloud', in classical noncommutative algebraic geometry of pi-algebras, as developed by Fred Van Oystaeyen and Alain Verschoren [23] in the early 80-ties.

Let $S$ be a finitely generated Noetherian $\mathbb{C}$-algebra satisfying all polynomial identities of degree $n$. Observe that $\int_{n} R, A_{n}$ and its subalgebras

$$
A_{c}=\operatorname{Im}(\beta) C \quad \text { and } \quad A_{e}=\operatorname{Im}(\beta) C_{A}(\operatorname{Im}(\beta))
$$

all satify these requirements. With $\operatorname{spec}(S)$ we denote the set of all twosided prime ideals of $S$ which becomes a topological space by equipping it with the Zariski topology having as typical closed sets $\mathbb{V}(I)=\{P \in \operatorname{spec}(S) \mid I \subset P\}$ for $I$ a twosided ideal of $S$. However, this twosided prime spectrum is not necessarily functorial, that is, if $S \stackrel{f}{\longrightarrow} T$ is a $\mathbb{C}$-algebra morphism between suitable algebras, then $f^{-1}(P)$ for a twosided prime ideal $P \triangleleft T$ does not have to be a prime ideal in $S$. Still, the twosided prime spectrum is functorial whenever $S \stackrel{f}{\longrightarrow} T$ is an extension, that is, if $T=\operatorname{Im}(f) C_{T}(\operatorname{Im}(f))$ by [17, Thm. II.6.5].

In 23, V.3] Van Oystaeyen and Verschoren constructed a noncommutative structure sheaf $\mathcal{O}_{S}^{b i}$ on $\operatorname{spec}(S)$ by means of localization in the category of $S$-bimodules. The main properties of this construction are 23, Thm. V.3.17 and Thm. V.3.36]

- One recovers the algebra back from the global sections $S=\Gamma\left(\operatorname{spec}(S), \mathcal{O}_{S}^{b i}\right)$

- Every extension $S \stackrel{f}{\longrightarrow} T$ defines a morphism of ringed spaces

$$
\left(\operatorname{spec}(T), \mathcal{O}_{T}^{b i}\right) \longrightarrow\left(\operatorname{spec}(S), \mathcal{O}_{S}^{b i}\right)
$$

In fact, the results are stated there only for prime Noetherian rings satisfying all polynomial identities of degree $n$ but extend to the case of interest here as our rings are finite modules over an affine center and hence we have enough central localizations. For more details, see [24].

An algebraic $D$-brane $R \stackrel{\beta}{\longrightarrow} A$ of degree $n$ is not necessarily an extension, but the morphisms below defined by it are :

$$
\int_{n} R \stackrel{\beta}{\longrightarrow} A_{c}=\operatorname{Im}(\beta) C \text { and } \int_{n} R \stackrel{\beta}{\longrightarrow} A_{e}=\operatorname{Im}(\beta) C_{A}(\operatorname{Im}(\beta))
$$

Theorem 3. Associate to the D-brane either of these noncommutative geometric data

$$
\left(\operatorname{spec}\left(A_{*}\right), \mathcal{O}_{A_{*}}^{b i}\right) \longrightarrow\left(\operatorname{spec}\left(\int_{n} R\right), \mathcal{O}_{\int_{n} R}^{b i}\right)
$$


It contains enough information to reconstruct $\beta: \int_{n} R \longrightarrow A$ by taking global sections, and hence by compositing with the universal morphism $R \longrightarrow \int_{n} R$ also the D-brane.

The fact that noncommutative prime spectra and bimodule structure sheaves behave only functorial with respect to $\mathbb{C}$-algebra morphisms which are extensions, explains the notion of a 'noncommutative cloud' in [9. In our language, the noncommutative cloud of a $C$-Azumaya algebra $A$ is the set

$$
\operatorname{cloud}(A)=\bigsqcup_{A_{*}} \operatorname{spec}\left(A_{*}\right)
$$

where the disjoint union of noncommutative prime spectra is taken over all subalgebras $A_{*}$ satisfying

$$
C \subset Z\left(A_{*}\right) \subset A_{*} \subset A
$$

and such subalgebras are called 'surrogates' in 9 . The point being that we can now associate to an algebraic D-brane of degree $n, R \stackrel{\beta}{\longrightarrow} A$ the partially defined map which is well defined because $\int_{n} R \longrightarrow A_{e}$ is an extension

$$
\operatorname{cloud}(A) \longleftrightarrow \operatorname{spec}\left(A_{e}\right) \longrightarrow \operatorname{spec}\left(\int_{n} R\right)
$$

and by adorning cloud $(A)$ componentswise with noncommutative structure sheaves $\mathcal{O}_{A_{*}}^{b i}$, this partially defined map contains enough information to reconstruct the Dbrane.

3.2. Noncommutative thin schemes. In [6, §I.2] Maxim Kontsevich and Yan Soibelman define a noncommutative thin scheme to be a covariant functor commuting with finite projective limits

$$
\mathrm{X}: \mathrm{fd}-\mathrm{alg} \longrightarrow \text { sets }
$$

from the category $f d-a l g$ of all finite dimensional $\mathbb{C}$-algebras to the category sets of all sets. They prove [6, Thm. 2.1.1] that every noncommutative thin scheme is represented by a $\mathbb{C}$-coalgebra. That is, there is a $\mathbb{C}$-coalgebra $C_{\mathrm{X}}$ associated to the noncommutative thin scheme $\mathrm{X}$ having the property that there is a natural one-to-one correspondence

$$
\mathrm{X}(B)=\operatorname{alg}\left(B, C_{\mathrm{X}}^{*}\right)
$$

for every finite dimensional $\mathbb{C}$-algebra $B$. Here, $C_{\mathrm{x}}^{*}$ is the dual algebra of all linear functionals on $C_{\mathrm{X}} . C_{\mathrm{X}}$ is called the coalgebra of distributions on $\mathrm{X}$ and the noncommutative algebra of functions on the thin scheme $\mathrm{X}$ is defined to be $\mathbb{C}[\mathrm{X}]=C_{\mathrm{X}}^{*}$. For a $\mathbb{C}$-algebra $R$, it is not true in general that the linear functionals $R^{*}$ are a coalgebra, but the dual coalgebra $R^{o}$ is, where

$$
R^{0}=\left\{f \in R^{*} \mid \operatorname{Ker}(f) \text { contains a twosided ideal of finite codimension }\right\}
$$

Kostant duality, see for example [21, Thm. 6.0.5], asserts that the functors

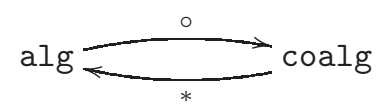

are adjoint. That is, for every $\mathbb{C}$-algebra $R$ and $\mathbb{C}$-coalgebra $C$ there is a natural one-to-one correspondence between the homomorphisms

$$
\operatorname{alg}\left(R, C^{*}\right)=\operatorname{coalg}\left(C, A^{\circ}\right)
$$


For an affine $\mathbb{C}$-algebra $R$ we define the contravariant functor

$$
\operatorname{rep}_{R}: \mathrm{fd} \text {-coalg } \longrightarrow \operatorname{sets} \quad C \mapsto \operatorname{alg}\left(R, C^{*}\right)
$$

describing the finite dimensional representations of $R$, see [6, Example 2.1.9]. As taking the linear dual restricts Koszul duality to an aniti-equivalence between the categories $f d-a l g$ and $f d$-coalg, we can describe $\operatorname{rep}_{R}$ as the noncommutative thin scheme represented by the dual coalgebra $R^{\circ}$ as

$$
\operatorname{rep}_{R}: \mathrm{fd}-\mathrm{alg} \longrightarrow \operatorname{sets} \quad B=C^{*} \mapsto \operatorname{alg}(R, B)=\operatorname{coalg}\left(C, R^{\circ}\right)
$$

Definition 5. The noncommutative affine scheme $\mathrm{rep}_{R}$ is the noncommutative thin scheme represented by the dual coalgebra $R^{\circ}$ of the affine $\mathbb{C}$-algebra $R$.

By [21, Lemma 6.0.1] for every $\mathbb{C}$-algebra morphism $f \in \operatorname{alg}(R, B)$, the dual map determines a $\mathbb{C}$-coalgebra morphism $f^{*} \in \operatorname{coalg}\left(B^{\circ}, R^{\circ}\right)$. In particular, to an algebraic D-brane $\beta: R \longrightarrow A$ we can associate a morphism between their noncommutative affine schemes

$$
\beta^{*}: \operatorname{rep}_{A} \longrightarrow \operatorname{rep}_{R}
$$

determined by the coalgebra map $\beta^{*}: A^{\circ} \longrightarrow R^{\circ}$.

Theorem 4. The morphism $\beta^{*}: \mathrm{rep}_{A} \longrightarrow \mathrm{rep}_{R}$ between the noncommutative thin schemes allows to reconstruct the algebraic D-brane $\beta: R \longrightarrow A$.

In order to prove this we need to describe the dual coalgebras $A^{o}$ and $R^{o}$. Recall that a coalgebra $D$ is said to be simple if it has no proper nontrivial sub-coalgebras. Every simple $\mathbb{C}$-coalgebra is finite dimensional and as $D^{*}$ is a simple $\mathbb{C}$-algebra, we have that $D \simeq M_{n}(\mathbb{C})^{*}$, the full matrix coalgebra that is, $\sum_{i, j} \mathbb{C} e_{i j}$ with

$$
\Delta\left(e_{i j}\right)=\sum_{k=1}^{n} e_{i k} \otimes e_{k j} \quad \text { and } \quad \epsilon\left(e_{i j}\right)=\delta_{i j}
$$

The coradical corad $(C)$ of a coalgebra $C$ is the direct sum of all simple subcoalgebras of $C$. It follows from Kostant duality that for any affine $\mathbb{C}$-algebra $R$ we have

$$
\operatorname{corad}\left(R^{o}\right)=\oplus_{S \in \operatorname{simp} R} M_{n}(\mathbb{C})_{S}^{*}
$$

where $\operatorname{simp} R$ is the set of isomorphism classes of finite dimensional simple $R$ representations and the factor of $\operatorname{corad}\left(R^{o}\right)$ corresponding to a simple representation $S$ is isomorphic to the matrix coalgebra $M_{n}(\mathbb{C})^{*}$ if $\operatorname{dim}(S)=n$.

In algebra, one can resize idempotents by Morita-theory and hence replace full matrices by the basefield. In coalgebra-theory there is an analogous duality known as Takeuchi equivalence, see 22. The isotypical decomposition of $\operatorname{corad}\left(R^{o}\right)$ as an $R^{o}$-comodule is of the form $\oplus_{S} C_{S}^{\oplus n_{S}}$, the sum again taken over all simple finite dimensional $R$-representations. Take the $R^{\circ}$-comodule $E=\oplus_{S} C_{S}$ and its coendomorphism coalgebra

$$
R^{\dagger}=\operatorname{coend}^{R^{o}}(E)
$$

then Takeuchi-equivalence (see for example [1, §4, §5] and the references contained in this paper for more details) asserts that $R^{o}$ is Takeuchi-equivalent to the coalgebra $R^{\dagger}$ which is pointed, that is, $\operatorname{corad}\left(R^{\dagger}\right)=\mathbb{C} \operatorname{simp}(R)=\oplus_{S} \mathbb{C} g_{S}$ with one group-like element $g_{S}$ for every simple finite dimensional $R$-representation. Remains to describe the structure of the full basic coalgebra $R^{\dagger}$. 
Example 2. For the affine Azumaya algebra $A$ of degree $n$ over its affine center $C$, we know that all finite dimensional simple $A$-representations are $n$-dimensional and are parametrized by the maximal ideals $\mathfrak{m} \in \max (C)$. That is, $\operatorname{corad}\left(A^{\circ}\right)=$ $\oplus_{\mathfrak{m}} M_{n}(\mathbb{C})^{*}$ and $A^{o}$ is Takeuchi-equivalent to the pointed coalgebra

$$
A^{\dagger}=C^{o} \quad \text { with } \operatorname{corad}\left(A^{\dagger}\right)=\oplus_{\mathfrak{m}} \mathbb{C} g_{\mathfrak{m}}
$$

By [21, Prop. 8.0.7] we know that any cocommutative pointed coalgebra is the direct sum of its pointed irreducible components (at the algebra level this says that a commutative semi-local algebra is the direct sum of local algebras). Therefore,

$$
A^{\dagger}=C^{0}=\oplus_{\mathfrak{m}} C_{\mathfrak{m}}^{0}
$$

where $C_{\mathfrak{m}}^{0}$ is a pointed irreducible cocommutative coalgebra and as such is a subcoalgebra of the enveloping coalgebra of the abelian Lie algebra on the Zariski tangent space $\left(\mathfrak{m} / \mathfrak{m}^{2}\right)^{*}$. That is, we recover the maximal spectrum $\max (C)$ of the center $C$ from $A^{\dagger}$. But then, the dual algebra

$$
A^{\dagger *}=C^{\circ *}=\prod_{\mathfrak{m}} \hat{C}_{\mathfrak{m}}
$$

the direct sum of the completions of $C$ at all maximal ideals $\mathfrak{m}$. Also, the double dual algebra

$$
A^{o *}=\prod_{\mathfrak{m}} M_{n}\left(\hat{C}_{\mathfrak{m}}\right)
$$

For a general affine noncommutative $\mathbb{C}$-algebra $R$, the description of the pointed coalgebra $R^{\dagger}$ is more complicated as there can be non-trivial extensions between non-isomorphic finite dimensional simple $R$-representations (note that this does not happen for Azumaya algebras).

For a (possibly infinite) quiver $\vec{Q}$ we define the path coalgebra $\mathbb{C} \vec{Q}$ to be the vectorspace $\oplus_{p} \mathbb{C} p$ with basis all oriented paths $p$ in the quiver $\vec{Q}$ (including those of length zero, corresponding to the vertices) and with structural maps induced by

$$
\Delta(p)=\sum_{p=p^{\prime} p^{\prime \prime}} p^{\prime} \otimes p^{\prime \prime} \quad \text { and } \quad \epsilon(p)=\delta_{0, l(p)}
$$

where $p^{\prime} p$ " denotes the concatenation of the oriented paths $p^{\prime}$ and $p^{\prime \prime}$ and where $l(p)$ denotes the length of the path $p$. Hence, every vertex $v$ is a group-like element and for an arrow (2) $\stackrel{a}{\longrightarrow}$ (a) we have $\Delta(a)=v \otimes a+a \otimes w$ and $\epsilon(a)=0$, that is, arrows are skew-primitive elements.

For every natural number $i$, we define the ext $t^{i}$-quiver $\overrightarrow{\operatorname{ext}}_{R}^{i}$ to have one vertex $v_{S}$ for every $S \in \operatorname{simp}(R)$ and such that the number of arrows from $v_{S}$ to $v_{T}$ is equal to the dimension of the space $\operatorname{Ext}_{R}^{i}(S, T)$. With $\operatorname{ext}_{R}^{i}$ we denote the $\mathbb{C}$-vectorspace on the arrows of $\overrightarrow{\operatorname{ext}}_{R}^{i}$.

The Yoneda-space $\operatorname{ext}_{R}^{\bullet}=\oplus \operatorname{ext}_{R}^{i}$ is endowed with a natural $A_{\infty}$-structure [4, defining a linear map (the homotopy Maurer-Cartan map, [20)

$$
\mu=\oplus_{i} m_{i}: \mathbb{C}_{\operatorname{ext}}^{1} \longrightarrow \operatorname{ext}_{R}^{2}
$$

from the path coalgebra $\overrightarrow{C e x t}_{R}^{1}$ of the $\operatorname{ext}^{1}$-quiver to the vectorspace $\operatorname{ext}_{R}^{2}$, see [4, $\S 2.2]$ and $[20$. 
Theorem 5. The dual coalgebra $R^{o}$ is Takeuchi-equivalent to the pointed coalgebra $R^{\dagger}$ which is the sum of all subcoalgebras contained in the kernel of the linear map

$$
\mu=\oplus_{i} m_{i}: \mathbb{C} \overrightarrow{\operatorname{ext}}_{R}^{1} \longrightarrow \operatorname{ext}_{R}^{2}
$$

determined by the $A_{\infty}$-structure on the Yoneda-space ext $_{R}^{\bullet}$.

Proof. We can reduce to finite subquivers as any subcoalgebra is the limit of finite dimensional subcoalgebras and because any finite dimensional $R$-representation involves only finitely many simples. Hence, the statement is a global version of the result on finite dimensional algebras due to B. Keller [4, §2.2].

Alternatively, we can use the results of E. Segal 20]. Let $S_{1}, \ldots, S_{r}$ be distinct simple finite dimensional $R$-representations and consider the semi-simple module $M=S_{1} \oplus \ldots \oplus S_{r}$ which determines an algebra epimorphism

$$
\pi_{M}: R \longrightarrow M_{n_{1}}(\mathbb{C}) \oplus \ldots \oplus M_{n_{r}}(\mathbb{C})=B
$$

If $\mathfrak{m}=\operatorname{Ker}\left(\pi_{M}\right)$, then the $\mathfrak{m}$-adic completion $\hat{R}_{\mathfrak{m}}=\underset{\leftarrow}{\lim } R / \mathfrak{m}^{n}$ is an augmented $B$-algebra and we are done if we can describe its finite dimensional (nilpotent) representations. Again, consider the $A_{\infty}$-structure on the Yoneda-algebra $\operatorname{Ext}_{R}^{\bullet}(M, M)$ and the quiver on $r$-vertices $\overrightarrow{\operatorname{ext}}_{R}^{1}(M, M)$ and the homotopy Mauer-Cartan map

$$
\mu_{M}=\oplus_{i} m_{i}: \mathbb{C e x t}_{R}^{1}(M, M) \longrightarrow \operatorname{Ext}_{R}^{2}(M, M)
$$

Dualizing we get a subspace $\operatorname{Im}\left(\mu_{M}^{*}\right)$ in the path-algebra $\mathbb{C} \overrightarrow{\operatorname{ext}}_{R}^{1}(M, M)^{*}$ of the dual quiver. Ed Segal's main result [20, Thm 2.12] now asserts that $\hat{R}_{\mathfrak{m}}$ is Moritaequivalent to

$$
\hat{R}_{\mathfrak{m}} \widetilde{M} \frac{\left(\overrightarrow{C e x t}_{R}^{1}(M, M)^{*}\right)^{\hat{M}}}{\left(\operatorname{Im}\left(\mu_{M}^{*}\right)\right)}
$$

where $\left(\mathbb{C}_{\operatorname{ext}}^{1}(M, M)^{*}\right)^{\wedge}$ is the completion of the path-algebra at the ideals generated by the paths of positive length. The statement above is the dual coalgebra version of this.

Proof of theorem 4: The morphism between the thin noncommutative schemes

$$
\beta^{*}: \operatorname{rep}_{A} \longrightarrow \operatorname{rep}_{R}
$$

corresponds to the coalgebra-map dual to $\beta$

$$
\beta^{o}: A^{o} \longrightarrow R^{o}
$$

Dualizing this map again we obtain a $\mathbb{C}$-algebra map, and composing with the natural map $R \longrightarrow R^{o *}$ and the observations of example 2 we obtain an algebra map

$$
R \longrightarrow R^{o *} \stackrel{\beta^{o *}}{\longrightarrow} A^{o *}=\prod_{\mathfrak{m}} M_{n}\left(\hat{C}_{\mathfrak{m}}\right)
$$

the components of which are the maps from the global sections of the structure sheaf of $R$ in the étale topology to the stalks of the structure sheaf of $A$ in the étale topology, induced by $\beta$. By étale descent we can therefore reconstruct the algebraic D-brane $\beta: R \longrightarrow A$ from them. 


\section{REFERENCES}

[1] William Chin, A brief introduction to coalgebra representation theory, in "Hopf Algebras" M. Dekker Lect. Notes in Pure and Appl. Math. (2004) 109-132. Online at http://condor.depaul.edu/ wchin/crt.pdf

[2] Aise Johan de Jong, The Stacks Project, Algebraic stacks - Examples

[3] Frank De Meyer and E. Ingraham, Separable algebras over commutative rings, Springer LNM $181(1970)$

[4] Bernhard Keller, A-infinity algebras in representation theory, Contribution to the Proceedings of ICRA IX, Beijing (2000). Online at http://www.math.jussieu.fr/ keller/publ/art.dvi

[5] Max-Albert Knus and Manuel Ojanguren, Théorie de la Descente et Algèbres d'Azumaya, Springer LNM 389 (1974)

[6] Maxim Kontsevich and Yan Soibelman, Notes on $A_{\infty}$-algebras, $A_{\infty}$-categories and noncommutative geometry I, arXiv:math.RA/0606241 (2006)

[7] Lieven Le Bruyn, Noncommutative geometry and Cayley-smooth orders, Pure and applied mathematics 290, Chapman \& Hall (2008)

[8] Lieven Le Bruyn and Stijn Symens, Partial desingularizations arising from non-commutative algebras, arXiv:0507494 (2005)

[9] Chien-Hao Liu and Shing-Tung Yau, Azumaya-type noncommutative spaces and morphisms therefrom: Polchinski's D-branes in string theory from Grothendieck's viewpoint, arXiv:0709.1515 (2007)

[10] Si Li, Chien-Hao Liu, Ruifang Song and Shing-Tung Yau, Morphisms from Azumaya prestable curves with a fundamental module to a projective variety: Topological D-strings as a master object for curves, arXiv:0809.2121 (2008)

[11] Chien-Hao Liu and Shing-Tung Yau, Azumaya structure on D-branes and resolution of ADE orbifold singularities revisited: Douglas-Moore vs. Polchinski-Grothendieck, arXiv:0901.0342 (2009)

[12] Chien-Hao Liu and Shing-Tung Yau, Azumaya structure on D-branes and deformations and resolutions of a conifold revisited: Klebanov-Strassler-Witten vs. Polchinski-Grothendieck, arXiv:0907.0268 (2009)

[13] Chien-Hao Liu and Shing-Tung Yau, Nontrivial Azumaya noncommutative schemes, morphisms therefrom, and their extension by the sheaf of algebras of differential operators: $D$ branes in a B-field background à la Polchinski-Grothendieck Ansatz, arXiv:0909.2291 (2009)

[14] Chien-Hao Liu and Shing-Tung Yau, D-branes and Azumaya noncommutative geometry: From Polchinski to Grothendieck, arXiv:1003.1178 (2010)

[15] Saunders Mac Lane, Categories for the working mathematician, Springer Graduate Texts in Math. 5, 2nd edition (1997)

[16] James S. Milne, Étale cohomology, Princeton Math. Series 33 (1980)

[17] Claudio Procesi, Rings with polynomial identities, Pure and Appl. Math. 17 Marcel Dekker (1973)

[18] Claudio Procesi, A formal inverse to the Cayley-Hamilton theorem, J. Alg. 107 (1987) 63-74

[19] Claudio Procesi, Deformations of representations, Methods in ring theory (Levico Terme, 1997), 247-276, Lecture Notes in Pure and Appl. Math. 198, Marcel Dekker (1998)

[20] Ed Segal, The $A_{\infty}$ deformation theory of a point and the derived category of local CalabiYaus, math.AG/0702539 (2007)

[21] Moss E. Sweedler, Hopf Algebras, monograph, W.A. Benjamin (New York) (1969)

[22] M. Takeuchi, Morita theorems for categories of comodules, J. Fac. Sci. Univ. Tokyo 24 (1977) 629-644

[23] Fred Van Oystaeyen and Alain Verschoren, Noncommutative algebraic geometry, Springer LNM 887 (1981)

[24] Fred Van Oystaeyen, Algebraic geometry for associative algebras, Lecture Notes in Pure and Appl. Math. 232 Marcel Dekker (2000)

Department of Mathematics, University of Antwerp, Middelheimlaan 1, B-2020 ANTWERP (BELGIUM), lieven.lebruyn@ua.ac.be 